\title{
Neutron-Antineutron Oscillation Matrix Elements with Domain Wall Fermions at the Physical Point
}

\author{
Sergey Syritsyn ${ }^{* a, b}$, Michael Buchoff ${ }^{c, d}$, Chris Schroeder ${ }^{c}$, Joe Wasem $^{c}$ \\ ${ }^{a}$ RIKEN/BNL Research Center, Brookhaven National Laboratory, Upton, NY 11973, USA \\ ${ }^{b}$ Jefferson Laboratory, 12000 Jefferson Ave, Newport News, VA 23606, USA \\ ${ }^{c}$ Lawrence Livermore National Laboratory, Livermore, California 94550, USA \\ ${ }^{d}$ Institute for Nuclear Theory, Box 351550, Seattle, WA 98195-1550, USA \\ E-mail: ssyritsyn@quark.phy.bnl.gov
}

\begin{abstract}
Neutron-antineutron oscillations are hypothetical processes changing the baryon number by 2 units. Compared to proton decay, they present another scenario of baryon number violation and, if found, they would have different implications for phenomenology of baryogenesis. If such baryon number violation exists at higher scales beyond the Standard model, it will manifest itself at the hadron scale as effective six-(anti)quark operators turning neutrons into antineutrons and vice versa. Nucleon matrix elements of these operators are important for experiments looking for evidence of such processes. We present preliminary results for these matrix elements computed with physical $N_{f}=2+1$ domain wall quarks. Results are non-perturbatively renormalized and converted to the $\overline{M S}$ scheme.
\end{abstract}

The 33rd International Symposium on Lattice Field Theory

14-18 July 2015

Kobe International Conference Center, Kobe, Japan

\footnotetext{
* Speaker.
} 


\section{Introduction}

One of the biggest puzzles in our current understanding of the early Universe is the baryon asymmetry (BAU), i.e. the excess of baryonic matter over antimatter. For such asymmetry to appear, the three famous Sakharov's conditions are required [1,2], one of which is that the baryon number is not conserved. Two kinds of phenomena, if observed, can demonstrate that the baryon number is violated. One is proton decay that has been sought in decades-long experiments such as (Super-)Kamiokande. The other is neutron-antineutron oscillation, that may also be connected to lepton number violations in unification models, e.g., see Ref. [3]. Neutron-antineutron oscillations change the baryon number by $|\Delta B|=2$ units and could potentially occur in vacuum or in nuclei. Experiments $[4,5,6,7]$ have set lower bounds on the oscillation time $\tau_{n \bar{n}} \gtrsim(1 \ldots 3) \cdot 10^{8} s$.

The effective $n \bar{n}$ Hamiltonian describing the oscillations has the form

$$
\mathscr{H}=\left(\begin{array}{cc}
E+V & \delta m \\
\delta m & E-V
\end{array}\right), \quad \delta m=-\sum_{i} c_{i}\left\langle\bar{n}\left|\mathscr{O}_{i}^{6 q}\right| n\right\rangle, \quad \tau_{n \bar{n}}=(2 \delta m)^{-1}
$$

where $E \pm V$ is the energy of the (anti)nucleon in the medium, $\tau_{n \bar{n}}$ is the ostillation time in vacuum, and $\mathscr{O}_{i}^{6 q}$ are effective 6-quark operators changing the baryon number by two units. These effective operators and their couplings $c_{i}$ to the quark sector must come from interactions beyond the Standard Model. Apart from the couplings, the $n \bar{n}$ oscillation rate depends on the matrix elements of the effective operators between (anti)nucleon states. Knowing these matrix elements is necessary to constrain extensions of the Standard Model in the context of baryogenesis.

Earlier calculations of these matrix elements were performed using various models such as MIT Bag model [8]. Some preliminary results have been reported from a lattice QCD calculation with Wilson quarks [9]. In this paper, we report preliminary results of complete lattice QCD calculation of the relevant $n \bar{n}$ matrix elements, including non-perturbative renormalization. This calculation was performed with dynamical chirally-symmetric quarks at the physical point using one value of the lattice volume and spacing.

\section{2. $N-\bar{N}$ matrix elements and lattice}

The effective six-quark operators that are required for neutron-antineutron transitions were classified in Ref.[10]. Since these operators are contributions to the Lagrangian density, they must be color- and electric charge-neutral (pseudo)scalars. Building these operators from chiral diquarks $\left(q^{T} C q\right)_{L, R}$ results in 18 operators $\left(C=i \gamma^{2} \gamma^{0}\right.$ is the charge conjugation matrix $\left.{ }^{1}\right)$ :

$$
\begin{aligned}
& \mathscr{O}_{\chi_{1}\left\{\chi_{2} \chi_{3}\right\}}^{1}=T_{i j k l m n}^{s}\left[u_{\chi_{1}}^{i T} C u_{\chi_{1}}^{j}\right]\left[d_{\chi_{2}}^{k T} C d_{\chi_{2}}^{l}\right]\left[d_{\chi_{3}}^{m T} C d_{\chi_{3}}^{n}\right], \\
& \mathscr{O}_{\left\{\chi_{1} \chi_{2}\right\} \chi_{3}}^{2}=T_{i j k l m n}^{s}\left[u_{\chi_{1}}^{i T} C d_{\chi_{1}}^{j}\right]\left[u_{\chi_{2}}^{k T} C d_{\chi_{2}}^{l}\right]\left[d_{\chi_{3}}^{m T} C d_{\chi_{3}}^{n}\right], \\
& \mathscr{O}_{\left\{\chi_{1} \chi_{2}\right\} \chi_{3}}^{3}=T_{i j k l m n}^{a}\left[u_{\chi_{1}}^{i T} C d_{\chi_{1}}^{j}\right]\left[u_{\chi_{2}}^{k T} C d_{\chi_{2}}^{l}\right]\left[d_{\chi_{3}}^{m T} C d_{\chi_{3}}^{n}\right],
\end{aligned}
$$

\footnotetext{
${ }^{1}$ In Minkowski $C$ is defined using Minkowski $\gamma$-matrices, $\left\{\gamma^{\mu}, \gamma^{\nu}\right\}=2 g^{\mu \nu}$, and on a Euclidean lattice we use $C_{\text {Euc }}=i \gamma_{\text {Euc }}^{2} \gamma_{\text {Euc }}^{4}$. Euclidean $\gamma$-matrices, $\left\{\gamma_{\mu}, \gamma_{v}\right\}=\delta_{\mu \nu},\left(\gamma_{\mu}\right)^{\dagger}=\gamma_{\mu}$, will be assumed for the rest of the manuscript.
} 
where $\chi_{1,2,3}=L, R$ and the color tensors $T^{s, a}$ are defined as

$$
\begin{aligned}
T_{i j k l m n}^{s} & =\varepsilon_{i k m} \varepsilon_{j l n}+\varepsilon_{j k m} \varepsilon_{i l n}+\varepsilon_{i l m} \varepsilon_{j k n}+\varepsilon_{j l m} \varepsilon_{i k n}, \\
T_{i j k l m n}^{a} & =\varepsilon_{i j m} \varepsilon_{k l n}+\varepsilon_{k l m} \varepsilon_{i j n},
\end{aligned}
$$

and the number is further reduced by Fierz transformation to 14 operators.

Table 1: Classification of $N-\bar{N}$ transition operators according to $S U(3)_{L, R}$ flavor symmetry. The other seven operators are obtained by replacing $L \leftrightarrow R$. The operators are built from left/right diquarks denoted as $L, R$, respectively, in the 1 st column. Notation $(\ldots)_{\mathbf{I}}$ denotes projection on representation with total isospin $I$. The 2nd column shows corresponding operators in terms of Eq.(2.1). The $S U(2)_{L, R}$ representation is shown in the 3rd column. The 1-loop anomalous dimension is given in the 4th column.

\begin{tabular}{l|l|c|c}
\hline \hline & $\mathscr{O}^{6 q}$ & $\mathbf{I}_{R} \otimes \mathbf{I}_{L}$ & $\gamma^{\mathscr{O}}$ \\
\hline$\left[(R R R)_{\mathbf{3}}\right]$ & $\mathscr{O}_{R(R R)}^{1}+4 \mathscr{O}_{(R R) R}^{2}$ & $\mathbf{3}_{R} \otimes \mathbf{0}_{L}$ & $\left(\alpha_{S} / 4 \pi\right)(-12)$ \\
\hline$\left[(R R R)_{\mathbf{1}}\right]$ & $\mathscr{O}_{(R R) R}^{2}-\mathscr{O}_{R(R R)}^{1} \equiv 3 \mathscr{O}_{(R R) R}^{3}$ & $\mathbf{1}_{R} \otimes \mathbf{0}_{L}$ & $\left(\alpha_{S} / 4 \pi\right)(-2)$ \\
{$\left[R_{\mathbf{1}}(L L)_{\mathbf{0}}\right]$} & $\mathscr{O}_{(L L) R}^{2}-\mathscr{O}_{L(L R)}^{1} \equiv 3 \mathscr{O}_{(L L) R}^{3}$ & $\mathbf{1}_{R} \otimes \mathbf{0}_{L}$ & 0 \\
{$\left[(R R)_{\mathbf{1}} L_{\mathbf{0}}\right]$} & $3 \mathscr{O}_{(L R) R}^{3}$ & $\mathbf{1}_{R} \otimes \mathbf{0}_{L}$ & $\left(\alpha_{S} / 4 \pi\right)(+2)$ \\
\hline$\left[(R R)_{2} L_{\mathbf{1}}\right]_{(1)}$ & $\mathscr{O}_{L(R R)}^{1}$ & $\mathbf{2}_{R} \otimes \mathbf{1}_{L}$ & $\left(\alpha_{S} / 4 \pi\right)(-6)$ \\
{$\left[(R R)_{\mathbf{2}} L_{\mathbf{1}}\right]_{(2)}$} & $\mathscr{O}_{(L R) R}^{2}$ & $\mathbf{2}_{R} \otimes \mathbf{1}_{L}$ & $\left(\alpha_{S} / 4 \pi\right)(-6)$ \\
{$\left[(R R)_{\mathbf{2}} L_{\mathbf{1}}\right]_{(3)}$} & $\mathscr{O}_{R(L R)}^{1}+2 \mathscr{O}_{(R R) L}^{2}$ & $\mathbf{2}_{R} \otimes \mathbf{1}_{L}$ & $\left(\alpha_{S} / 4 \pi\right)(-6)$ \\
\hline \hline
\end{tabular}

In order to further proceed with operator classification, it is useful to convert them to $S U(2)_{L} \otimes$ $S U(2)_{R}$ chiral isospin basis, see Tab. 1. In this basis, the perturbative renormalization is diagonal [11]. Note that if the total $S U(2)_{L+R}$ isospin of an operator is greater than 1, the matrix elements between neutron and antineutron states are identically zero, e.g for $\left[(L L L)_{\mathbf{3}}\right]$ and $\left[(R R R)_{\mathbf{3}}\right]$ (line 1 in Tab. 1). Further restrictions arise from the electroweak symmetry: the operators must be singlets under the $S U(2)_{L} \times U(1)_{Y}$ gauge group (which also implies zero electric charge). Therefore, we are left with only six operators, of which three are independent and symmetric under the electroweak symmetry (lines 2-4 in Tab. 1), and the other three are not electroweak-symmetric and connected to each other by isospin symmetry (lines 5-7 in Tab. 1). We evaluate matrix elements of all six operators to verify our methodology.
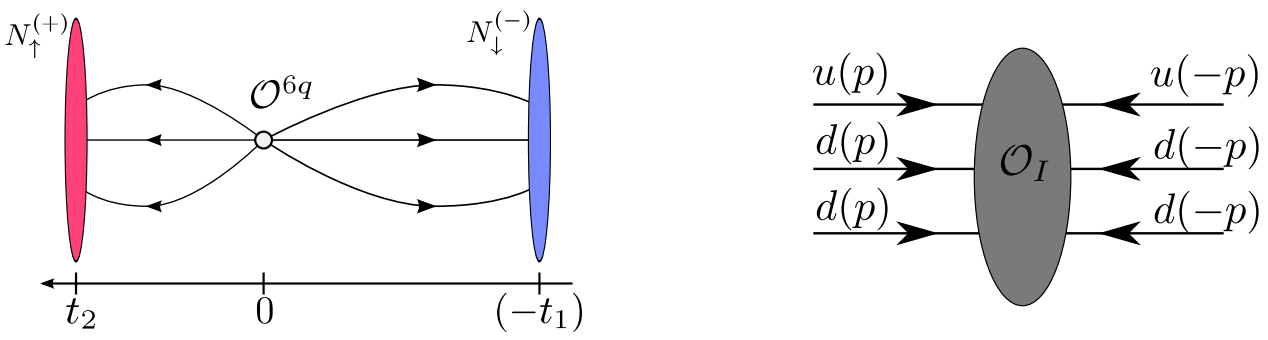

Figure 1: (Left) Contractions for three-point functions of 6-quark operators and the neutron. (Right) Assignment of momenta in Green's function for non-perturbative renormalization on a lattice (RI-MOM), before averaging over the momenta permutations.

For our calculation of the $n \bar{n}$ transition matrix elements we use gauge configurations with $N_{f}=$ $2+1$ dynamical Möbius domain wall fermions generated by the UKQCD/RBC collaborations[12]. 
Chiral symmetry of the fermions is important for simple multiplicative renormalization of operators $^{2}$. These gauge configurations correspond to physical pion mass $m_{\pi} \approx 140 \mathrm{MeV}$ and lattice spacing $a=0.114 \mathrm{fm}$ and have volume $48^{3} \times 96$. The spatial size corresponds to $m_{\pi} L \approx 3.9$ and we expect that finite volume effects are small.

Lattice contractions for three-point functions involving 6-quark operators

$$
C_{n \mathscr{O} \bar{n}}\left(t_{2}, 0,-t_{1}\right)=\left\langle N_{\uparrow}^{(+)}\left(t_{2}\right) \mathscr{O}(0) N_{\downarrow}^{(-)}\left(-t_{1}\right)\right\rangle
$$

are shown in Fig.1(Left): an antineutron with spin $s_{z}=+\frac{1}{2}$ is created with negative-parity nucleon interpolating field $N_{\downarrow}^{(-)}$at timeslice $-t_{1}<0$, and a neutron with $s_{z}=+\frac{1}{2}$ is annihilated with positive-parity operator $N_{\uparrow}^{(+)}$at timeslice $t_{2}>0$. There are no disconnected diagrams. An economical computing method is to calculate point-source propagators starting at the location of the operator and contract them with with neutron and antineutron interpolating fields. In addition, such scheme provides all combinations of time separations between the operator and the nuncleon interpolating fields, allowing detailed analysis of excited state contaminations. Neutron operators are built from Gaussian-smeared quark fields with parameters used for nucleon structure calculations [13]. In this preliminary analysis of nucleon matrix elements we compute a simple ratio of three- to two-point functions that cancels the exponential factors,

$$
\left\langle n\left|\mathscr{O}^{6 q}\right| \bar{n}\right\rangle=\frac{C_{n \mathscr{O} \bar{n}}^{S S}\left(t_{2}, 0,-t_{1}\right)}{C_{n n}^{P S}\left(t_{2}+t_{1}\right)} \cdot \frac{C^{P P}\left(t^{*}\right)}{C^{P S}\left(t^{*}\right)},
$$

where "S" stands for smeared and "P" stands for point quark sources (1st) and sinks (2nd). The second ratio cancels neutron normalization factors ratio $\sqrt{Z_{N}^{S} / Z_{N}^{P}}$, which we evaluate at $t^{*}=t_{1}+t_{2}$ in this preliminary analysis. The time separation $t^{*}$ may be increased to avoid excited states in the $C_{n n}^{P P}$ two-point function, although at $t^{*} \geq 10 a$ they are already negligible (see Fig.2).

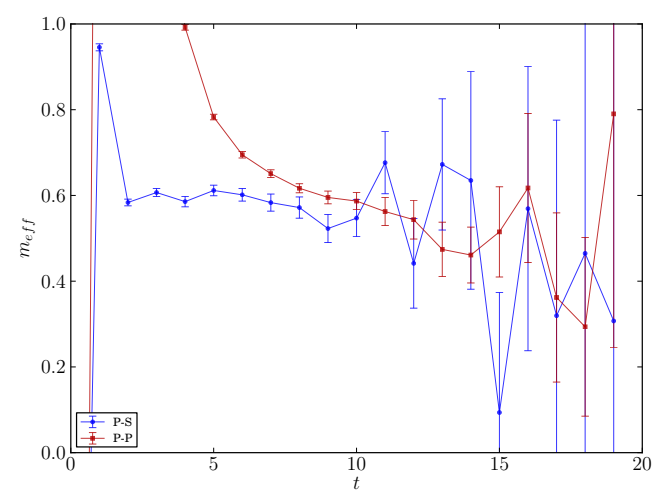

Figure 2: Effective mass in the $C_{n n}^{P S}(t)$ and $C_{n n}^{P P}(t)$ two-point correlation functions. Their ratio is used to cancel the smeared and unsmeared nucleon normalization factors.

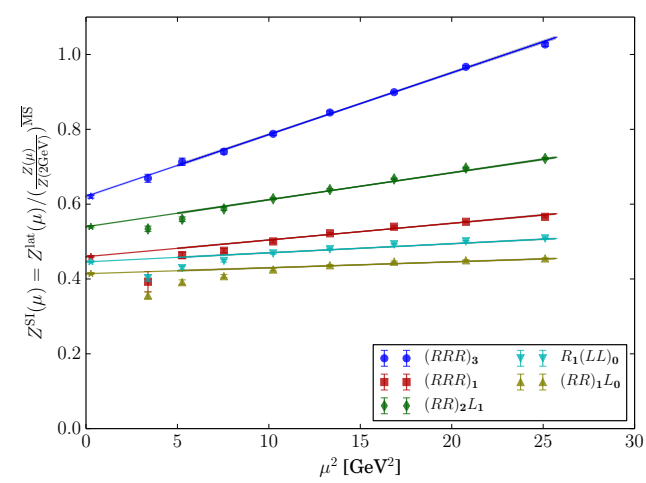

Figure 3: Scale-independent renormalization factors in the momentum-shell scheme shown in Fig. 1(right) and Eq. 3.1.

\footnotetext{
${ }^{2}$ Earlier calculations [9] used Wilson fermions. We did not compute renormalization factors on those ensembles. In a theory with explicit chiral symmetry breaking one would have to switch from the chiral to the parity basis of operators.
} 
In Figure 4 we show the correlator ratios (2.5) that correspond to matrix elements of bare sixquark operators vs. the source-sink separation $T=t_{1}+t_{2}=6 \ldots 12$ and the operator insetion time $\tau=t_{o p}-\frac{1}{2}\left(t_{2}-t_{1}\right)$. At the current statistical precision, there are no visible effects from excited states above $T \geq 10 a$, and we select plateau values at $T=10 a$ as our estimate of the matrix elements in this preliminary analysis.
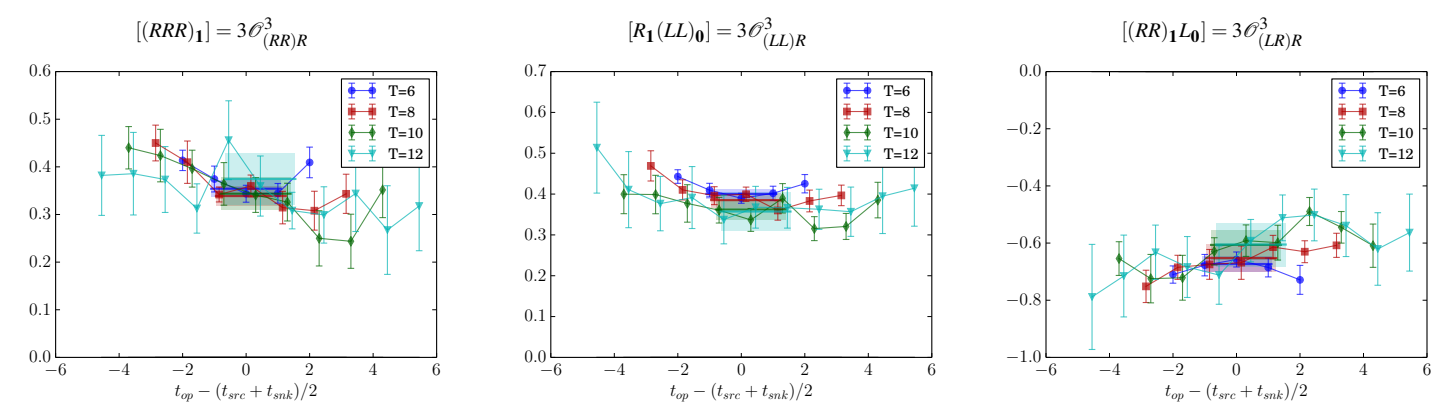

$\left[(R R)_{2} L_{1}\right]^{(1)}=\mathscr{O}_{(L R) R}^{1}$
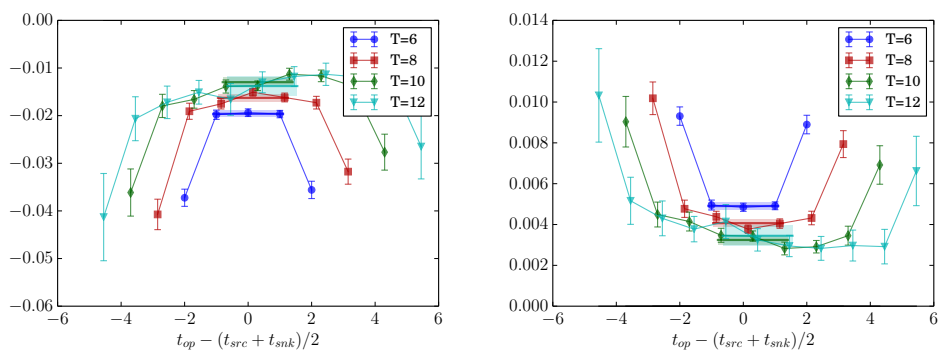

$\left[(R R)_{2} L_{\mathbf{1}}\right]^{(3)}=\mathscr{O}_{R(R L)}^{1}+2 \mathscr{O}_{(R R) L}^{2}$

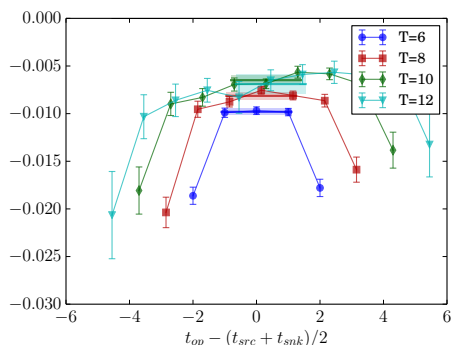

Figure 4: Plateaus of ratios of bare three-point to two-point correlators, scaled by $10^{6}$. The renormalization, nucleon opeorator overlap, and $a^{6}$ factors has not been applied.

\section{Nonperturbative Renormalization}

The six-quark operator matrix elements computed on a lattice must be renormalized to a scheme used in pertrubative calculations, such as $\overline{M S}(2 \mathrm{GeV})$. In this preliminary work, we perform match between lattice and continuum using 1-loop perturbative calculation [11 $]^{3}$.

We compute lattice quark-operator Green's functions in Landau gauge. Only quark fields carry momentuma $\pm p$, while the operator momentum is zero. There is no need to assign non-zero momentum $\sim|p|$ to the operator, because there are no antiquark fields in the correlator and no poles can appear in the chiral limit; also, such momentum arrangement would complicate perturbative calculations. Since the total momentum of the six quark fields has to cancel, three quarks carry momentum $p$ and the other three carry $(-p)$ (see Fig. 1(right)). Unfortunately, such assignment breaks the symmetry of the vertex functions under $S U(2)_{R, L}$ transformations, since $(u(p), d(-p))_{R, L}$ is not an isospin doublet, and leads to mixing of vertex functions from different multiplets both on a lattice and in perturbation theory. One can project the vertex functions onto multiplets explicitly to make RI-MOM renormalization diagonal in isospin multiplets. However, the simpler and more intuitive trick is to "restore" the isospin symmetry by averaging the Green's function over all possible

\footnotetext{
${ }^{3}$ At the moment, 2-loop renormalization is available, with 1-loop matching coefficients between momentum-shell and $\overline{M S}$ schemes $[9,14]$, which will be used in a subsequent publication.
} 
momentum permutations,

$$
\begin{aligned}
G_{i j k l m n}^{I, 6 q}\left(p^{2}\right) & =\frac{1}{5}\left\langle\mathscr{O}^{I, 6 q} u_{i}(+p) u_{j}(+p) d_{k}(+p) d_{l}(-p) d_{m}(-p) d_{n}(-p)\right\rangle \\
& +\frac{3}{5}\left\langle\mathscr{O}^{I, 6 q} u_{i}(+p) u_{j}(-p) d_{k}(+p) d_{l}(+p) d_{m}(-p) d_{n}(-p)\right\rangle \\
& +\frac{1}{5}\left\langle\mathscr{O}^{I, 6 q} u_{i}(-p) u_{j}(-p) d_{k}(+p) d_{l}(+p) d_{m}(+p) d_{n}(-p)\right\rangle,
\end{aligned}
$$

where the coefficients are combinatorial and result from counting permutations. Since such permutation effectively restores the isospin symmetry broken by non-equal momenta, the operator $\mathscr{O}^{I, 6 q}$ and its Green's function $G^{I, 6 q}(p)$ in Eq.(3.1) transform as the same isospin multiplet $I$ under $S U(2)_{R, L}$.

We select the lattice momenta along the 4-dimensional diagonals $( \pm 1, \pm 1, \pm 1, \pm 1)$, which are expected to have the least amount of discretization effects. The renormalization factors for six-quark operators are extracted from lattice Green's functions of the operators,

$$
Z_{\mathscr{O}^{6 q}}=\left(Z_{A} \cdot \frac{\Lambda_{A}^{\text {tree }}}{Z_{A}^{\text {lat }}}\right)^{3} \cdot\left(\frac{\Lambda_{\mathscr{O}^{6 q}}^{\text {tree }}}{\Lambda_{\mathscr{O}^{6 q}}^{\text {lat }}}\right)
$$

where the first factor cancels out the quark field renormalization $Z_{q}^{3}$ from the second factor and $Z_{A}$ is extracted from the local to conserved axial current [12]. Using the 1-loop running of $\Delta B=2$ six-quark operators [11], we plot scale-independent renormalization constants normalized for $\mu=$ $2 \mathrm{GeV}$ for a range of lattice momenta $p^{2}$ in Fig. 3. Lattice scaling for some of the operators is clearly not described by 1-loop perturbative calculations, which is most likely caused by very large $O\left(p^{2}\right)$ discretization effects. The two representations with largest scaling deviations are $\left(3_{R}, 0_{L}\right)$, $\left(2_{R}, 1_{L}\right)$, and their $R \leftrightarrow L$ counterparts. Fortunately, these operators are not $S U(2)_{L}$-symmetric and do not have consequences for phenomenology. We extract the renormalization factor from quadratic fits $Z(p) \sim Z+\alpha a^{2} p^{2}$ in ranges $p=2 \ldots 4$ and $4 \ldots 6 \mathrm{GeV}$; we take their average as the central value and half of their difference as the systematic error of the renormalization factors. To be conservative, we take the variance in the region $p=4 \ldots 6 \mathrm{GeV}$ as the systematic error for renormalization of the operators $\left(3_{R}, 0_{L}\right)$ and $\left(2_{R}, 1_{L}\right)$, which have the largest discretization error.

\section{Results and discussion}

The results for the $n \bar{n}$ matrix elements of the six-quark operators are renormalized to $\overline{M S}(2 \mathrm{GeV})$ and summarized in Tab. 2. In addition, we show the comparison of our results to the bag model [8]. In the cases relevant for the phenomenology, we see that ab initio calculations yield matrix elements $\times(5 \ldots 8)$ larger than model calculations. This difference must provide tighter constraints on BSM models, which we will discuss in the upcoming publication.

Although these results should be treated as preliminary, they are obtained with chiral fermions at the physical point, with substantial evidence that the nucleon ground state dominates the computed matrix elements. Together with the nonperturbative renormalization, these results are very likely to remove any theoretical uncertainties from the nucleon structure in the phenomenology of the $n \bar{n}$ oscillations. 
Table 2: Preliminarly results for matrix elements of 6-quark operators 2.1 and comparison to the MIT Bag Model results [8]. The first line shows matrix elements for $I=3_{R, L}$ operators vanishing identically.

\begin{tabular}{l|rr|rr|rr}
\hline \hline & $Z($ lat $\rightarrow \overline{M S})$ & $\mathscr{O}^{\overline{M S}(2 \mathrm{GeV})}\left[10^{-5} \mathrm{GeV}^{6}\right]$ & Bag “A” & $\frac{L Q C D}{\text { Bag "A" }}$ & Bag "B" & $\frac{L Q C D}{\text { Bag "B" }}$ \\
\hline$\left[(R R R)_{\mathbf{3}}\right]$ & $0.62(12)$ & 0 & 0 & - & 0 & - \\
\hline$\left[(R R R)_{\mathbf{1}}\right]$ & $0.454(33)$ & $45.4(5.6)$ & 8.190 & 5.5 & 6.660 & 6.8 \\
{$\left[R_{\mathbf{1}}(L L)_{\mathbf{0}}\right]$} & $0.435(26)$ & $44.0(4.1)$ & 7.230 & 6.1 & 6.090 & 7.2 \\
{$\left[(R R)_{\mathbf{1}} L_{\mathbf{0}}\right]$} & $0.396(31)$ & $-66.6(7.7)$ & -9.540 & 7.0 & -8.160 & 8.1 \\
\hline$\left[(R R)_{\mathbf{2}} L_{\mathbf{1}}\right]^{(1)}$ & $0.537(52)$ & $-2.12(26)$ & 1.260 & -1.7 & -0.666 & 3.2 \\
{$\left[(R R)_{\mathbf{2}} L_{\mathbf{1}}\right]^{(2)}$} & $0.537(52)$ & $0.531(64)$ & -0.314 & -1.7 & 0.167 & 3.2 \\
{$\left[(R R)_{\mathbf{2}} L_{\mathbf{1}}\right]^{(3)}$} & $0.537(52)$ & $-1.06(13)$ & 0.630 & -1.7 & -0.330 & 3.2 \\
\hline \hline
\end{tabular}

\section{ACKNOWLEDGEMENTS}

We thank the RBC and UKQCD collaborations for providing us with the gauge configurations. Computations for this work were carried out on facilities of the USQCD Collaboration, which are funded by the Office of Science of the U.S. Department of Energy, and on Lawrence Livermore National Laboratory computing clusters, operated under the auspices of the U.S. Department of Energy by Lawrence Livermore National Security, LLC, Lawrence Livermore National Laboratory under Contract DE-AC52-07NA27344. This work was supported by RIKEN Foreign Postdoctoral Researcher Program and by Nathan Isgur Fellowship program at the Jefferson National Laboratory (SNS).

\section{References}

[1] A. Sakharov, Pisma Zh.Eksp.Teor.Fiz. 5, 32 (1967).

[2] V. A. Kuzmin, Pisma Zh. Eksp. Teor. Fiz. 12, 335 (1970).

[3] R. N. Mohapatra and R. Marshak, Phys.Rev.Lett. 44, 1316 (1980).

[4] M. Baldo-Ceolin et al., Z.Phys. C63, 409 (1994).

[5] J. Chung et al., Phys. Rev. D66, 032004 (2002).

[6] K. Abe et al., Phys. Rev. D91, 072006 (2015).

[7] M. Bergevin, Search for Neutron Anti-Neutron Oscillation at the Sudbury Neutrino Observatory, $\mathrm{PhD}$ thesis, University of Guelph, 2011.

[8] S. Rao and R. Shrock, Phys.Lett. B116, 238 (1982).

[9] M. I. Buchoff, C. Schroeder, and J. Wasem, (2012).

[10] T.-K. Kuo and S. Love, Phys.Rev.Lett. 45, 93 (1980).

[11] W. E. Caswell, J. Milutinovic, and G. Senjanovic, Phys.Lett. B122, 373 (1983).

[12] T. Blum et al., Phys. Rev. D93, 074505 (2016).

[13] S. Syritsyn et al., PoS LATTICE2014, 134 (2015).

[14] M. I. Buchoff and M. Wagman, Phys. Rev. D93, 016005 (2016). 